\title{
Cosmological-parameter determination with microwave background maps
}

\author{
Gerard Jungman* \\ Department of Physics, Syracuse University, Syracuse, New York 13244 \\ Marc Kamionkowski ${ }^{\dagger}$ \\ Department of Physics, Columbia University, New York, New York 10027 \\ Arthur Kosowsky \\ Harvard-Smithsonian Center for Astrophysics, 60 Garden Street, Cambridge, Massachusetts 02138 \\ and Department of Physics, Lyman Laboratory, Harvard University, Cambridge, Massachusetts 02138 \\ David N. Spergel ${ }^{\S}$ \\ Department of Astrophysical Sciences, Princeton University, Princeton, New Jersey 08544 \\ and Department of Astronomy, University of Maryland, College Park, Maryland 20742
}

(Received 20 December 1995)

\begin{abstract}
The angular power spectrum of the cosmic microwave background (CMB) contains information on virtually all cosmological parameters of interest, including the geometry of the Universe $(\Omega)$, the baryon density, the Hubble constant $(h)$, the cosmological constant $(\Lambda)$, the number of light neutrinos, the ionization history, and the amplitudes and spectral indices of the primordial scalar and tensor perturbation spectra. We review the imprint of each parameter on the CMB. Assuming only that the primordial perturbations were adiabatic, we use a covariance-matrix approach to estimate the precision with which these parameters can be determined by a CMB temperature map as a function of the fraction of sky mapped, the level of pixel noise, and the angular resolution. For example, with no prior information about any of the cosmological parameters, a full-sky CMB map with $0.5^{\circ}$ angular resolution and a noise level of $15 \mu \mathrm{K}$ per pixel can determine $\Omega, h$, and $\Lambda$ with standard errors of \pm 0.1 or better, and provide determinations of other parameters which are inaccessible with traditional observations. Smaller beam sizes or prior information on some of the other parameters from other observations improves the sensitivity. The dependence on the underlying cosmological model is discussed. [S0556-2821(96)02014-0]

PACS number(s): 98.70.Vc, 98.80.Bp, 98.80.Es
\end{abstract}

\section{INTRODUCTION}

One of the fundamental goals of observational cosmology today is measurement of the classical cosmological parameters: the total density (or, equivalently, the geometry) of the Universe, $\Omega$; the cosmological constant $\Lambda$; the baryon density $\Omega_{b}$; and the Hubble constant $H_{0}$. Accurate measurement of these quantities will test the cornerstones of the hot big-bang theory and will provide answers to some of the outstanding questions in cosmology. For example, determination of the geometry of the Universe will tell us the ultimate fate of the Universe and test the inflationary paradigm, while an independent check of $\Omega_{b}$ can confirm the predictions of big-bang nucleosynthesis.

In addition, parameters describing primordial perturbations are related to the origin of large-scale structure in the Universe and may shed light on a possible inflationary epoch. Perhaps the most important of these are the normalization $Q_{S}$ and spectral index $n_{S}$ of the primordial spectrum of scalar perturbations that gave rise to the observed structure.

\footnotetext{
*Electronic address:jungman@npac.syr.edu

†Electronic address: kamion@phys.columbia.edu

*Electronic address: akosowsky@cfa.harvard.edu

${ }^{\S}$ Electronic address: dns@astro.princeton.edu
}

Inflation may produce a spectrum of gravity waves, quantified by an amplitude $Q_{T}$ and spectral index $n_{T}$. A neutrino species with a mass greater than $1 \mathrm{eV}$ affects structure formation, and so the number $N_{\nu}$ of light (meaning $m_{\nu} \lesssim 1 \mathrm{eV}$ ) neutrinos is another cosmological parameter of importance. The ionization history of the Universe is also certainly related to the evolution of structure in the Universe.

In this paper, we estimate how well cosmological parameters can be determined from a cosmic microwave background (CMB) temperature map. Since the initial detection of temperature anisotropies in the CMB by the Cosmic Background Explorer (COBE) satellite [1], over a dozen other balloon-borne and ground-based experiments have announced anisotropy detections on smaller angular scales [2]. With the existence of anisotropies now firmly established, sights are shifting to accurate determination of the CMB power spectrum over a wide range of angular scales. Several technological advances, including improved amplifiers, interferometry, and long-duration balloon flights, hold great promise for high-precision measurements. Ultimately, a satellite with subdegree angular resolution will provide a detailed map of the entire microwave sky in multiple frequency bands [3].

A detailed map of the cosmic microwave background can potentially provide a wealth of information on the values of cosmological parameters. Roughly speaking, the amount of 
information in a map is proportional to the number of pixels on the sky, and this is inversely proportional to the square of the beam width. Thus, a map with a beam width of $0.5^{\circ}$ will contain over 100 times as much information as COBE, which had a beam width of order $7^{\circ}$, and an $0.1^{\circ}$-resolution experiment would have, roughly speaking, $10^{4}$ times as much information. It should be no surprise, therefore, that a map with good angular resolution should be able to determine many more cosmological parameters than COBE, which really only constrains the normalization of the $\mathrm{CMB}$ power spectrum and the effective CMB spectral index at large angular scales.

We consider an experiment which maps a given fraction of the sky with a given angular resolution and a given level of pixel noise. We use a covariance-matrix approach to evaluate the standard errors which would arise by fitting the power spectrum obtained in this experiment to all the unknown cosmological parameters. We display results for a range of realistic values for the fraction of sky covered, level of pixel noise, and angular resolution. Our results are quite promising: With minimal assumptions, realistic satellite experiments could potentially determine $\Omega, \Lambda$, and the inflationary observables to far greater precision than any traditional measurements. Furthermore, the information provided on other parameters will be competitive with (and with additional reasonable assumptions, superior to) current probes. Although we focus here only on models with primordial adiabatic perturbations, we are confident that if the perturbations turn out to be isocurvature, it will be evident in the temperature maps (and perhaps also in polarization maps, spectral distortions, and non-Gaussian temperature distributions), and that similar results on parameter determination will apply. Indeed, recent calculations of the CMB power spectrum in defect models [4] and in isocurvature models [5] suggest that such models should be clearly distinguishable from the adiabatic case. Although we have satellite mapping experiments in mind, our results can also be applied to ground or balloon experiments, or to the combined results of several complementary measurements.

An important issue facing any likelihood analysis is the choice of the space of models considered. Here we consider models with primordial adiabatic perturbations. Our space of models allows a cosmological constant, an open (or closed) Universe, tensor modes (with a free spectral index), variations in the baryon density and Hubble constant, tilted primordial spectra, and primordial spectra that deviate from pure power laws. We assume that the dark matter is cold; however, the CMB power spectrum is only slightly altered in mixed and hot-dark-matter models [6], and we allow the number of massless neutrinos to vary. Therefore, our conclusions on parameter determination will be virtually independent of the fraction of hot dark matter.

In the following section, we describe our calculation of the power spectrum. In Sec. III, we illustrate the effect of each cosmological parameter that we consider on the CMB spectrum. In Sec. IV, we discuss the covariance matrix. To illustrate, in Sec. V, we present results for the standard errors to the parameters that would be obtained assuming the true cosmological model is standard cold dark matter (CDM). We also discuss how these results change if the underlying model differs from the canonical standard-CDM model. In
Sec. VI, we discuss the validity of the covariance-matrix approach to the analysis. In Sec. VII, we make some concluding remarks and discuss some future areas of investigation.

\section{CALCULATION OF THE CMB SPECTRUM}

In many areas of astrophysics, it is difficult to make detailed quantitative predictions as properties of complex systems depend on the nonlinear physics of poorly measured and poorly understood phenomena. Fortunately, the early Universe was very simple and nearly uniform. The density fluctuations are all in the linear regime $\left(\delta \rho / \rho \sim 10^{-4}\right)$ and nonlinear effects are unimportant. Different groups using different gauge choices and numerical algorithms make very similar predictions for CMB fluctuations for a given model. This simple linearity makes possible the detailed parameter determination that we describe in this paper.

The CMB angular power spectrum $C(\theta)$ is defined as

$$
C(\theta) \equiv\left\langle\frac{\Delta T}{T_{0}}(\hat{\mathbf{m}}) \frac{\Delta T}{T_{0}}(\hat{\mathbf{n}})\right\rangle, \quad \hat{\mathbf{m}} \cdot \hat{\mathbf{n}}=\cos \theta,
$$

where the angular brackets represent an ensemble average over all angles and observer positions. Here $\Delta T(\hat{\mathbf{n}}) / T_{0}$ is the fractional temperature fluctuation in the direction $\hat{\mathbf{n}}$, and the mean CMB temperature is $T_{0}=2.726 \pm 0.010 \mathrm{~K}$ [7]. This power spectrum is conveniently expressed in terms of its multipole moments $C_{l}$, defined by expanding the angular dependence in Legendre polynomials, $P_{l}(x)$ :

$$
C(\theta)=\sum_{l=2}^{\infty} \frac{2 l+1}{4 \pi} C_{l} P_{l}(\cos \theta) .
$$

Given a model for structure formation, calculation of the multipole moments is straightforward and is accomplished by solution of the coupled system of Boltzmann equations for each particle species (i.e., photons, baryons, massless and possibly massive neutrinos, and cold dark matter) and Einstein equations for the evolution of the metric perturbations. The $l=1$ term is indistinguishable from the Doppler shift due to proper motion with respect to the microwave background rest frame and is conventionally ignored. For theories with Gaussian initial perturbations, the set of $C_{l}$ completely specifies the statistical properties of the theory. Since we can only observe from a single vantage point in the Universe, the observed multipole moments $C_{l}^{\text {obs }}$ will be distributed about the mean value $C_{l}$ with a "cosmic variance" $\sigma_{l} \simeq \sqrt{2 /(2 l+1)} C_{l}$; no measurement can defeat this variance. Power-spectrum predictions and measurements are traditionally plotted as $l(l+1) C_{l}$ versus $l$.

For the purposes of covariance-matrix evaluation, as well as for likelihood maximization [8] and Monte Carlo analysis, it is useful to have an algorithm for rapid evaluation of the CMB spectrum for a given set of cosmological parameters. We begin with a semianalytic solution of the coupled Boltzmann, fluid, and Einstein equations developed by $\mathrm{Hu}$ and Sugiyama [9] for flat cold-dark-matter models, which we generalize to accommodate an open Universe, a cosmological constant, tensor modes, and reionization. The code is fast enough to enable likelihood analyses requiring tens of 
thousands of power-spectrum evaluations. We have checked that our semianalytic calculation agrees with the results of a publicly available numerical code [10] for several parameters. Here we briefly describe our calculation.

The multipole moments are expressed as

$$
C_{l}=C_{l}^{S}+C_{l}^{T},
$$

where $C_{l}^{S}$ is the contribution from scalar perturbations and $C_{l}^{T}$ is the contribution from tensor modes. The scalar contribution is given by

$$
C_{l}^{S}=\frac{2}{\pi} \int_{0}^{\infty} d k k^{2}\left|\Theta_{l}\left(\eta_{0}, k\right)\right|^{2},
$$

where $\eta_{0}$ is the conformal time today (the conformal time $\eta=\int d t / a$ with $a$ the scale factor of the Universe normalized to unity at matter-radiation equality). The contribution of wave number $k$ to the $l$ th multipole moment is [9]

$$
\begin{aligned}
\Theta_{l}\left(\eta_{0}, k\right) \simeq & {\left[\Theta_{0}+\Psi\right]\left(k, \eta_{*}\right) j_{l}\left(k \eta_{0}-k \eta_{*}\right) } \\
& +\Theta_{1}\left(k, \eta_{*}\right) j_{l}^{\prime}\left(k \eta_{0}-k \eta_{*}\right) \\
& +\int_{\eta_{*}}^{\eta_{0}} d \eta[\dot{\Psi}-\dot{\Phi}] j_{l}\left(k \eta_{0}-k \eta\right),
\end{aligned}
$$

where $\Theta_{0}$ and $\Theta_{1}$ are the monopole and dipole perturbations of the photon distribution function, $\Phi$ and $\Psi$ are gravitational-potential perturbations in the Newtonian gauge, $j_{l}$ are spherical Bessel functions and $j_{l}^{\prime}$ their first derivatives, and an overdot denotes a derivative with respect to conformal time. Here $\eta_{*}$ is the conformal time at decoupling. (See Ref. [9] for more details.) The third term in this expression gives the integrated Sachs-Wolfe (ISW) effect: Anisotropies are generated by time variations in the gravitational potentials along the line-of-sight path. Analytic fits to the gravitational potentials are given in Ref. [9], as are WKB solutions for the photon distributions in the tight-coupling regime, $\hat{\Theta}_{0}$ and $\hat{\Theta}_{1}$. At decoupling, photon diffusion (Silk damping) damps photon perturbations on small angular scales [11]; the perturbations to the photon distribution functions are given by $\left[\Theta_{0}+\Psi\right]\left(\eta_{*}\right)=\left[\hat{\Theta}_{0}+\Psi\right]\left(\eta_{*}\right) \mathcal{D}(k)$, where the mean damping factor is

$$
\mathcal{D}(k)=\int_{0}^{\eta_{0}} \dot{\tau} e^{-\left[k / k_{D}(\eta)\right]^{2}} d \eta .
$$

Here $\dot{\tau}=x_{e} n_{e} \sigma_{T} a / a_{0}$ is the differential optical depth for Thomson scattering, $n_{e}$ is the electron density, $x_{e}$ is the ionization fraction, and $\sigma_{T}$ is the Thomson cross section. The visibility function-the combination $\dot{\tau} e^{-\tau}$-is the probability density that a photon last scattered at given conformal time, and is sharply peaked near the surface of last scatter; semianalytic fits are given in Ref. [9]. As pointed out in Ref. [12], photon polarization must be included to obtain the proper Silk-damping scale; the result is

$$
k_{D}^{-2}(\eta)=\frac{1}{6} \int_{0}^{\eta} d \eta \frac{1}{\dot{\tau}} \frac{R^{2}+16(1+R) / 15}{(1+R)^{2}},
$$

where

$$
R=\frac{3 \rho_{b}}{4 \rho_{\gamma}}=\frac{3 \Omega_{b} a}{4\left(1-f_{\nu}\right) \Omega_{0}}
$$

is the scale factor normalized to $3 / 4$ at baryon-radiation equality, with $\Omega_{b}$ the fraction of critical density in baryons, $\Omega_{0}$ the fraction of critical density in nonrelativistic matter (baryons and cold dark matter), and $f_{\nu}$ the fraction of the total radiation density contributed by massless neutrinos. Our numerical evaluation of these expressions reproduces the power spectrum obtained from Boltzmann codes to an accuracy of a few percent for standard CDM.

Analytic approximations to the CMB anisotropy due to tensor modes (gravity waves) are given in Refs. [13,14]. The contribution to each multipole moment of the CMB power spectrum is

$$
C_{l}^{T}=36 \pi^{2} \frac{(l+2) !}{(l-2) !} \int_{0}^{\infty} d k P_{T}(k)\left|F_{l}(k)\right|^{2},
$$

where $P_{T} \propto k^{n_{T}+4}$ is the initial power spectrum of tensor perturbations and $F_{l}$ is given by

$$
\begin{aligned}
F_{l}(k) \equiv & k^{-3 / 2} \int_{\eta_{*}}^{\eta_{0}} d \eta \eta\left\{[1-w(\eta)] T\left(\frac{k}{k_{\mathrm{eq}}}, \eta\right) \frac{j_{2}(k \eta)}{(k \eta)^{2}}\right. \\
& \left.+w(\eta) \frac{j_{1}(k \eta)}{3 k \eta}\right\} \frac{j_{l}\left(k \eta_{0}-k \eta\right)}{\left(k \eta_{0}-k \eta\right)^{2}},
\end{aligned}
$$

with $k_{\text {eq }}$ defined as the wave number of the mode which enters the horizon at matter-radiation equality. The fitting function $w(\eta)$ describes the evolution of the gravity-wave mode function through the transition between the radiationdominated and matter-dominated epochs, and $T(k, \eta)$ is a transfer function describing the evolution of the tensor-mode amplitude. Good analytic fits to these two functions are given by $[14]$

$$
\begin{gathered}
w(\eta)=\exp \left(-0.2 \eta^{0.55}\right) \\
T(y, \eta)=\frac{\eta^{2}}{a}\left[e^{-4 y^{4}}\left(1+1.34 y+2.5 y^{2}\right)^{1 / 2}+1-e^{-4 y^{4}}\right] .
\end{gathered}
$$

These approximations match numerical results to $1 \%$ well past $l=100$, where the tensor contribution to the multipoles drops to a small fraction of the scalar contribution.

Equations (4) and (9) are difficult to evaluate numerically because of the oscillatory spherical Bessel functions in the integrand. Asymptotic expansions, a Bessel-function cache, and various interpolation techniques further speed evaluation of the integrals. We calculate every 40th multipole (more for $l<100$ ) and perform a cubic spline to recover the entire spectrum.

We consider models which are well described by a power-law spectrum of metric perturbations over the range of scales affecting CMB anisotropies. This class includes all inflation models. For the scalar perturbations, we also allow a deviation from power-law behavior and parametrize the power spectrum as [15] 


$$
P(k) \propto\left(\frac{k}{k_{S}}\right)^{n_{S}+\alpha \ln \left(k / k_{S}\right)},
$$

where $k_{S}$ is the normalization scale at which the power law index $n_{S}$ is defined. The parameter $\alpha$ quantifies the deviation from the power law or the "running" of the spectral index. Realistic inflation models can produce values of $\alpha$ large enough to change the multipole moments by as much as $5 \%$. For the tensor spectrum, we assume a pure power-law spectrum with spectral index $n_{T}$. In principle, $n_{T}$ can run with scale as well, but because of the comparatively small amount of information contained in the tensor multipole moments, the CMB constraint on the index $n_{T}$ is weak, and the running-index effect for the tensor perturbations is negligible.

Extensions of this basic cosmological model are incorporated through various fitting formulas. In a cosmologicalconstant $(\Lambda)$ Universe, the gravitational potential $\Phi$ begins to vary at low redshift when the Universe becomes cosmological-constant dominated, and this leads to a contribution to the anisotropy at large angles from the ISW effect. In a flat Universe (that is, $\Omega_{0}+\Lambda=1$, where $\Lambda$ is the cosmological constant in units of critical density), this is approximated by multiplying the multipole moments by a factor $[1+g(\Lambda) / l][16,17]$, where

$$
\begin{gathered}
g(\Lambda)=36 \pi \int_{0}^{\eta_{0}} \frac{1}{[F(0)]^{2}}\left(\frac{d F}{d \eta}\right)^{2}\left(\eta_{0}-\eta\right) d \eta, \\
F(\eta)=\frac{H}{a} \int \frac{d a / a_{0}}{\left(H a / a_{0}\right)^{3}}
\end{gathered}
$$

is the time dependence of the potential, and $H=\dot{a} / a$ is the Hubble parameter. This approximation slightly overestimates the lowest few multipole moments, but this large-angle ISW effect is generally not a large fraction of the total anisotropy, and the lowest multipole moments have a limited statistical significance. For $\Lambda \lesssim 0.7, g(\Lambda)$ can be approximated by

$$
g(\Lambda) \simeq 0.637\left(\frac{\Lambda}{1-\Lambda}\right)^{0.817} .
$$

An additional effect of a cosmological constant is a shift in the conformal distance to the surface of last scatter (even with the mass density $\Omega_{0} h^{2}$ held fixed), which we account for by multiplying the current conformal time $\eta_{0}$ by the correction factor $1+0.085 \ln (1-\Lambda)[18]$.

Generalization to an open Universe is somewhat more complicated because several different effects contribute to the anisotropy [17]. The angular scale subtended by the horizon at the surface of last scatter scales as $\Omega^{1 / 2}$ where $\Omega=\Omega_{0}+\Lambda$ is the total density (in units of critical density) of the Universe [19]. Therefore, the multipole moments in an open Universe are related to those in a flat Universe approximately by $D_{l}(\Omega) \simeq D_{l \Omega^{1 / 2}}(\Omega=1)$ with $D_{l}=l(l+1) C_{l}$. In other words, the CMB spectrum in an open Universe resembles that in a flat Universe with the same matter density, but shifted to smaller angular scales. A large-angle ISW effect arises from the evolution of the gravitational potentials, although the function $g(\Omega)$ differs from that in a cosmological-constant Universe [17]. In addition, the lowest multipole moments probe scales comparable to or larger than the curvature scale, and so these moments are suppressed, due heuristically to the exponential growth of volume in an open Universe at large distances. Finally, some ambiguity exists as to the correct generalization of a power-law spectrum to an open Universe. Naive power laws of volume, wave number, or eigenvalue of the Laplace operator differ in an open Universe [17], as do spectra predicted by various open-Universe inflationary scenarios [20]. However, these power laws differ only in their predictions for the lowest multipole moments, which have little statistical weight; for definiteness, we use the predictions of a specific inflationary scenario [21]. A good fit to these effects (for $\Omega \gtrsim 0.1$ ) is provided by multiplying the multipole moments by

$$
1+e^{-0.3 / / l_{\operatorname{curv}}} \frac{g(\Omega)}{l+1 / 2},
$$

where $l_{\text {curv }}=\pi \sqrt{(1-\Omega) / \Omega}$ is the multipole corresponding to the curvature scale of the Universe, and

$$
g(\Omega) \simeq 4.5\left(\frac{1-\Omega}{\Omega}\right)^{0.817},
$$

for $\Omega \gtrsim 0.1$.

If the Universe has experienced significant reionization between recombination and today, then a fraction $1-e^{-\tau_{\text {reion }}}$ of the CMB photons has scattered since recombination, where $\tau_{\text {reion }}$ is the optical depth to the epoch of recombination. If the Universe becomes reionized at a redshift $z_{\text {reion }}$ with a constant ionization fraction $x_{e}$, then the optical depth is $\tau_{\text {reion }} \simeq 0.04 \Omega_{b} h \Omega^{-1 / 2} x_{e}\left[\left(1+z_{\text {reion }}\right)^{3 / 2}-1\right]$, where $h$ is the Hubble parameter in units of $100 \mathrm{~km} \mathrm{sec}^{-1}$ $\mathrm{Mpc}^{-1}$. The precise effects of reionization depend on the baryon density, Hubble parameter, and the ionization history. However, as illustrated in Ref. [19] (see Fig. 3 therein), the effects of reionization are fairly accurately quantified solely in terms of $\tau_{\text {reion }}$. Compton scattering is an isotropizing process, and so the multipole moments on angular scales smaller than those subtended by the horizon at the epoch of reion-

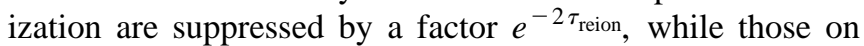
larger angular scales are unaffected. We interpolate between the asymptotic effects of reionization on small and large angular scales by multiplying the multipole moments by

$$
\exp \left[\frac{-2 \tau_{\text {reion }}\left(l \eta_{\text {reion }} / \eta_{0}\right)^{2}}{1+\left(l \eta_{\text {reion }} / \eta_{0}\right)^{2}}\right],
$$

where $\eta_{\text {reion }}$ is the conformal time at reionization. In addition, reionization also induces a broad Doppler peak centered near $l \simeq \eta_{0} / \eta_{\text {reion }}[22,23]$, but this secondary peak is shallow and we do not include it in the power-spectrum calculation.

Between the surface of last scatter and the present, several other physical processes, besides reionization, produce new CMB fluctuations and smear out primordial fluctuations [24]: gravitational lensing lowers the amplitude of the spectral peaks and fills in the valleys in the spectrum [25], the nonlinear growth of structure produces additional small-scale fluctuations [26], the scattering of photons off of hot gas in clusters and superclusters produces both thermal and non- 
thermal cosmic microwave fluctuations [27,29], and secondorder effects in a reionized Universe also produce additional small-scale fluctuations [28,29]. These nonlinear effects are relatively small and typically produce only $\sim 1 \mu \mathrm{K}$ changes in the microwave multipoles. However, they are systematic. If they are not included in an analysis of a full-sky CMB map, they will lead to systematic errors in parameter estimation. We do not include these effects in our sensitivity analyses as they are unlikely to alter the size and shape of the error ellipsoid. It will be important to include these effects in any analysis of a future all-sky CMB map.

\section{COSMOLOGICAL PARAMETERS AND THE CMB SPECTRUM}

The suite of cosmological models that we consider all make broadly similar predictions for the CMB spectrum: The fluctuations on large angular scales are nearly scale invariant and are primarily due to large-scale variations in the gravitational potential at the surface of last scatter, while on small scales the fluctuations are primarily due to variations in the velocity and density of the baryon-photon fluid at the surface of last scatter. The details of the spectrum, however, depend sensitively on properties of the Universe: its geometry, its size, the baryon density, the matter density, and the shape of the primordial fluctuation spectrum. In this section, we discuss each parameter that we consider and illustrate its most salient effect on the CMB spectrum. Figure 1 illustrates the following discussion.

The first Doppler peak occurs at the angular scale subtended by the sonic horizon at the surface of last scatter. Since the photon energy density exceeds the baryon energy density at that epoch, the sound speed of the Universe is close to $c / \sqrt{3}$, so that the sonic horizon corresponds to a nearly fixed physical scale. The angular scale subtended by this fixed physical scale will depend on the geometry of the Universe. In an open Universe, the angular scale subtended by an object of fixed diameter at fixed large redshift scales as $\Omega$. On the other hand, the causal horizon at last scatter is actually $\Omega^{-1 / 2}$ times as large in an open Universe as it is in a flat Universe. Thus, to a first approximation, the flatUniverse CMB spectrum is stretched by a factor $\Omega^{1 / 2}$ to smaller angular scales in an open Universe.

Increasing the baryon density $\Omega_{b} h^{2}$ reduces the pressure at the surface of last scatter and therefore increases the anisotropy at the surface of last scatter. This reduction in pressure also lowers the sound speed of the baryon-photon fluid, which alters the location and spacing of the Doppler peaks. Increasing the matter density $\Omega_{0} h^{2}$ shifts matter-radiation equality to a higher redshift. This reduces the early-ISW contribution to the spectrum and lowers and narrows the first Doppler peak. If we knew that $\Lambda=0$, then the combination of these three effects (pressure, sound speed, and redshift of matter-radiation equality) would be sufficient to enable a determination of $\Omega_{0}, \Omega_{b}$, and $h$ from the CMB spectrum.

The cosmological constant introduces a near degeneracy in parameter determination. Bond et al. [30] stressed that the CMB spectrum changed little if $\Lambda$ was varied while $\Omega_{0} h^{2}$ and $\Omega_{b} h^{2}$ were held fixed in a flat Universe. Changing $\Lambda$, however, does alter the size of the Universe. The conformal
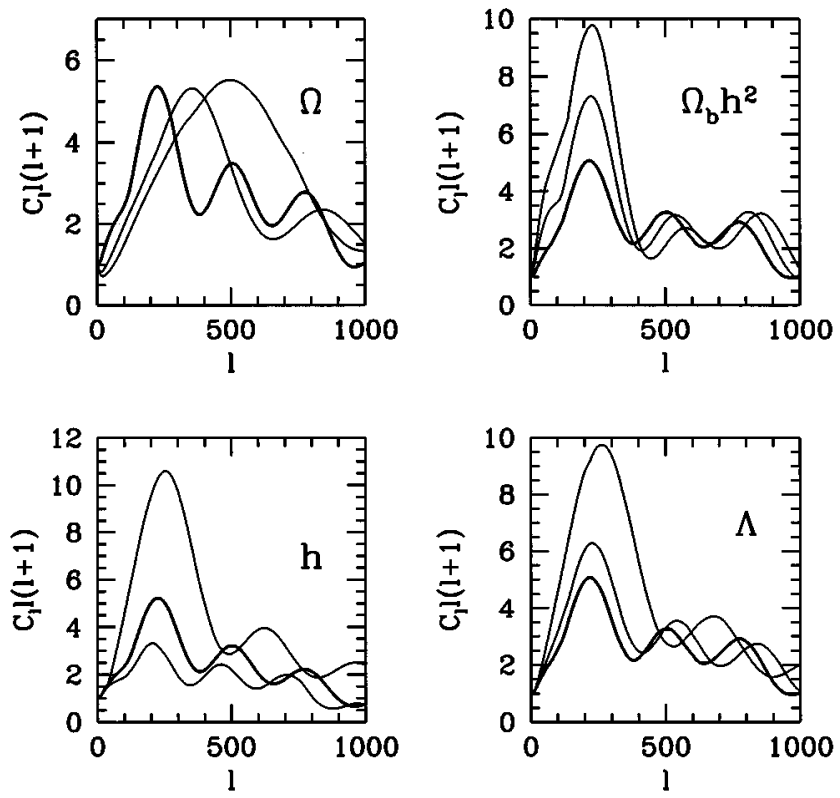

FIG. 1. Predicted multipole moments for standard CDM and variants. The heavy curves in each graph are for a model with primordial adiabatic perturbations with $\Omega=1, \Lambda=0, n_{S}=1$, $\Omega_{b} h^{2}=0.01, h=0.5, \alpha=0$, and no tensor modes. The graphs show the effects of varying $\Omega, \Lambda, h, \tau_{\text {reion }}=0$, and $\Omega_{b} h^{2}$ while holding all other parameters fixed. In the $\Omega$ panel, from left to right, the solid curves are for $\Omega=1, \Omega=0.5$, and $\Omega=0.3$. The curves in the $\Omega_{b} h^{2}$ panel are (from lower to upper) for $\Omega_{b} h^{2}=0.01$, $\Omega_{b} h^{2}=0.03$, and $\Omega_{b} h^{2}=0.05$. In the $h$ panel, the heavy curves is for $h=0.5$, while the other two curves are for $h=0.3$ (the upper light curve) and $h=0.7$ (the lower light curve). The curves in the $\Lambda$ panel are for (from lower to upper) $\Lambda=0, \Lambda=0.3$, and $\Lambda=0.7$.

distance from the present back to the surface of last scatter is smaller in a $\Lambda$-dominated flat Universe than in a matterdominated flat Universe. Thus, increasing $\Lambda$ shifts the Doppler peak to larger angular scales, the opposite effect of lower $\Omega_{0}$. This effect, along with the late-time ISW effect induced by $\Lambda$, breaks the degeneracy and enables an independent determination of all of the cosmological parameters directly from an all-sky high-resolution CMB map.

The value of $N_{\nu}$, the effective number of noninteracting relativistic degrees of freedom (in standard CDM, this is equal to three for the three light-neutrino species), also shifts the epoch of matter-radiation equality and thus the height of the first Doppler peak as discussed above. In addition, if $N_{\nu}$ is changed, the value of the anisotropic stress at early times-before the Universe is fully matter dominated-is altered, and this has a slight effect on the ISW contribution to the rise of the first Doppler peak.

The tensor-mode contribution to the multipole moments simply adds in quadrature with the scalar-mode contribution since there is no phase correlation between them. The amplitude of the tensor modes is parametrized by $r=Q_{T}^{2} / Q_{S}^{2}$, the ratio of the squares of the tensor and scalar contributions to the quadrupole moment. ${ }^{1}$ The index $n_{T}$ is defined so that the tensor-mode spectrum is roughly flat at large angular scales

${ }^{1}$ Note that this definition differs from that in Ref. [31]. 
for $n_{T}=0$; it falls steeply near the rise to the first Doppler peak. Thus, tensor modes may contribute to the anisotropy at large scales, but they will have little or no effect on the structure of the Doppler peaks. Increasing the tensor spectral index, $n_{T}$, increases the contribution at small angular scales relative to those at larger angles.

The overall normalization $Q$ raises or lowers the spectrum uniformly. The effect of the scalar spectral index is similarly simple: if $n_{S}$ is increased there is more power on small scales and vice versa. The effects of $\alpha$ are obvious from Eq. (13). Finally, the effects of reionization have been discussed in the previous section.

\section{ERROR ESTIMATES}

We consider an experiment which maps a fraction $f_{\text {sky }}$ of the sky with a Gaussian beam with full width at half maximum $\theta_{\text {FWHM }}$ and a pixel noise $\sigma_{\text {pix }}=s / \sqrt{t_{\text {pix }}}$, where $s$ is the detector sensitivity and $t_{\mathrm{pix}}$ is the time spent observing each $\theta_{\text {FWHM }} \times \theta_{\text {FWHM }}$ pixel. We adopt the inverse weight per solid angle, $w^{-1} \equiv\left(\sigma_{\text {pix }} \theta_{\mathrm{FWHM}} / T_{0}\right)$, as a measure of noise that is pixel-size independent [32]. Current state-of-the-art detectors achieve sensitivities of $s=200 \mu \mathrm{K} \sqrt{\mathrm{sec}}$, corresponding to an inverse weight of $w^{-1} \simeq 2 \times 10^{-15}$ for a 1 -year experiment. Realistically, however, foregrounds and other systematic effects may increase the effective noise level; conservatively, $w^{-1}$ will likely fall in the range $(0.9-4) \times 10^{-14}$. Treating the pixel noise as Gaussian and ignoring any correlations between pixels, estimates of $C_{l}$ can be approximated as normal distributions with a standard error $[32,33]$

$$
\sigma_{l}=\left[\frac{2}{(2 l+1) f_{\mathrm{sky}}}\right]^{1 / 2}\left[C_{l}+w^{-1} e^{l^{2} \sigma_{b}^{2}}\right],
$$

where $\sigma_{b}=7.42 \times 10^{-3}\left(\theta_{\mathrm{FWHM}} / 1^{\circ}\right)$. Note that Eq. (20) applies only if the entire sky has been mapped and then a fraction $1-f_{\text {sky }}$ has been subtracted. On the other hand, if only a fraction $f_{\text {sky }}$ of the sky is mapped, then the integration time per pixel increases by a factor of $f_{\text {sky }}^{-1}$, and $w^{-1}$ should be replaced by $w^{-1} f_{\text {sky }}$ [33].

In Fig. 2, we show simulated data that might be obtained with a CMB mapping experiment, given an underlying cosmological model of "standard CDM" (see the following section). The "cosmic variance" panel illustrates the multipole moments that would be measured by an ideal experiment (i.e., perfect angular resolution and no pixel noise); the scatter is due only to cosmic variance. The top-right and bottom-left panels show multipole moments that might be measured by full-sky mapping experiments with a realistic level of pixel noise and angular resolutions of $0.1^{\circ}$ and $0.3^{\circ}$, respectively. The cosmic variance slightly increases the errors at lower $l$, while the finite beam width is evident in the increased noise at $(l / 700) \geq\left(\theta_{\mathrm{FWHM}} / 0.3^{\circ}\right)^{-1}$ in the lower-left plot. The lower-right panel shows the moments from the lower-left panel after the total signal is smoothed with a Gaussian window of width $l / 20$. This illustrates that although the individual moments may be quite noisy, an experiment with a beam width of $0.3^{\circ}$ can still use the information in the location and shape of the third peak in parameter estimation. An experiment with this size beam can extract useful information out to $l \sim 900$, although it cannot
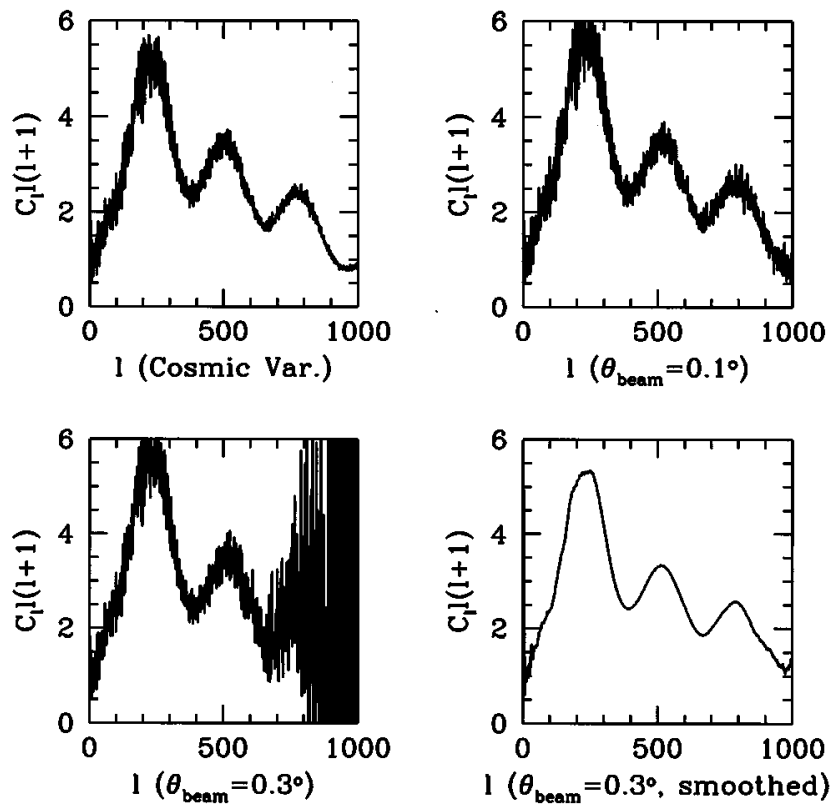

FIG. 2. Simulated data that might be obtained with a CMB mapping experiment, for beam sizes of $0.3^{\circ}$ and $0.1^{\circ}$, and a noise level of $w^{-1}=2 \times 10^{-15}$.

accurately measure the individual values of these high $l$ multipoles. The smoothing here is used for display and is not the optimal approach for parameter estimation.

We now wish to determine the precision with which a given CMB temperature map will be able to determine the various cosmological parameters. The answer to this question will depend not only on the experimental arrangement, but also on the correct underlying cosmological parameters which we seek to determine. For any given set of cosmological parameters, $\mathbf{s}=\left\{\Omega, \Omega_{b} h^{2}, h, \Lambda, n_{S}, r, n_{T}, \alpha, \tau_{\text {reion }}, Q, N_{\nu}\right\}$, the multipole moments, $C_{l}(\mathbf{s})$, can be calculated as described above. Suppose that the true parameters which describe the Universe are $\mathbf{s}_{0}$. If the probability for observing each multipole moment, $C_{l}^{\text {obs }}$, is nearly a Gaussian centered at $C_{l}$ with standard error $\sigma_{l}$, and $\theta_{\mathrm{FWHM}} \ll 1$ so that the largest multipole moments sampled are $l \gg 1$, then the probability distribution for observing a CMB power spectrum which is best fit by the parameters $\mathbf{S}$ is $[31,34,24]$

$$
P(\mathbf{s}) \propto \exp \left[-\frac{1}{2}\left(\mathbf{s}-\mathbf{s}_{0}\right) \cdot[\alpha] \cdot\left(\mathbf{s}-\mathbf{s}_{0}\right)\right],
$$

where the curvature matrix $[\alpha]$ is given approximately by

$$
\alpha_{i j}=\sum_{l} \frac{1}{\sigma_{l}^{2}}\left[\frac{\partial C_{l}\left(\mathbf{s}_{0}\right)}{\partial s_{i}} \frac{\partial C_{l}\left(\mathbf{s}_{0}\right)}{\partial s_{j}}\right] .
$$

As discussed in Ref. [31], the covariance matrix $[\mathcal{C}]=[\alpha]^{-1}$ gives an estimate of the standard errors that would be obtained from a maximum-likelihood fit to data: The standard error in measuring the parameter $s_{i}$ (obtained by integrating over all the other parameters) is approximately $\mathcal{C}_{i i}^{1 / 2}$. Prior information about the values of some of the parameters, from other observations or by assumption, is easily included. In the simplest case, if some of the parameters are known, then the covariance matrix for the others is deter- 
mined by inverting the submatrix of the undetermined parameters. For example, if all parameters are fixed except for $s_{i}$, the standard error in $s_{i}$ is simply $\alpha_{i i}^{-1 / 2}$.

Previous authors have investigated the sensitivity of a given experimental configuration to some small subset of the parameters we investigate here. For example, Knox investigated the sensitivity of mapping experiments to the inflationary parameters, $n_{S}, n_{T}$, and $r$, but assumed all other parameters (including $\Omega_{b}$ and $h$ ) were known [32]. Similarly, Hinshaw, Bennett, and Kogut investigated the sensitivity to $\Omega_{b}$ assuming all other parameters were fixed [35]. These were Monte Carlo studies which mapped the peak of the likelihood function. Another technique is to repeatedly simulate an experimental measurement of a given underlying theory, maximize the likelihood in each case, and see how well the underlying parameters are reproduced [8]. Such calculations require numerous evaluations of the CMB spectrum, and so the results have been limited to a small range of experimental configurations. If any of these analyses are limited to a small subset of cosmological parameters, they do not investigate the possible correlation with other undetermined parameters and will therefore overestimate the capability of the experiment to measure the parameters under consideration.

The covariance-matrix approach has the advantage that numerous experimental configurations and correlations between all the unknown cosmological parameters can be investigated with minimal computational effort. For example, if there are $N$ undetermined parameters, then we need only $N+1$ evaluations of the $C_{l}$ 's to calculate the partial derivatives in Eq. (21). Once these are evaluated, the curvature matrix for any combination of $w^{-1}$ and $\theta_{\mathrm{FWHM}}$ for $f_{\text {sky }}=1$ can be obtained trivially. The results are generalized to $f_{\text {sky }}<1$ by multiplying the results for the curvature matrix by $f_{\text {sky }}^{-1}$ [cf., Eqs. (20) and (22)]. Furthermore, the covariance matrix includes all correlations between parameters. Therefore, our results reproduce and generalize those in Refs. $[32,35,8]$, and we comment on this further below.

\section{COVARIANCE-MATRIX RESULTS}

As discussed above, the sensitivity of a CMB map to cosmological parameters will depend not only on the experiment, but also on the underlying parameters themselves. For illustration, we show results for a range of experimental parameters under the assumption that the underlying cosmological parameters take on the "standard-CDM" values, $\mathbf{s}_{0}=\left\{1,0.01,0.5,0,1,0,0,0,0, Q_{\mathrm{COBE}}, 3\right\}$, where $Q_{\mathrm{COBE}}=20 \mu \mathrm{K}$ is the COBE normalization [36]. (It assumes a HarrisonZeldovich primordial spectrum, no tensor modes, no cosmological constant, a flat Universe, and the central big-bang nucleosynthesis value for the baryon-to-photon ratio.) After presenting results for this assumed cosmological model, we briefly discuss how the results will be altered for different cosmological models.

With the 11 undetermined cosmological parameters we survey here, some of which are better determined by experiment than others, there is an endless number of combinations that could conceivably be investigated. Instead of running through all possible permutations, we present results for the standard errors that can be obtained with two extreme sets of

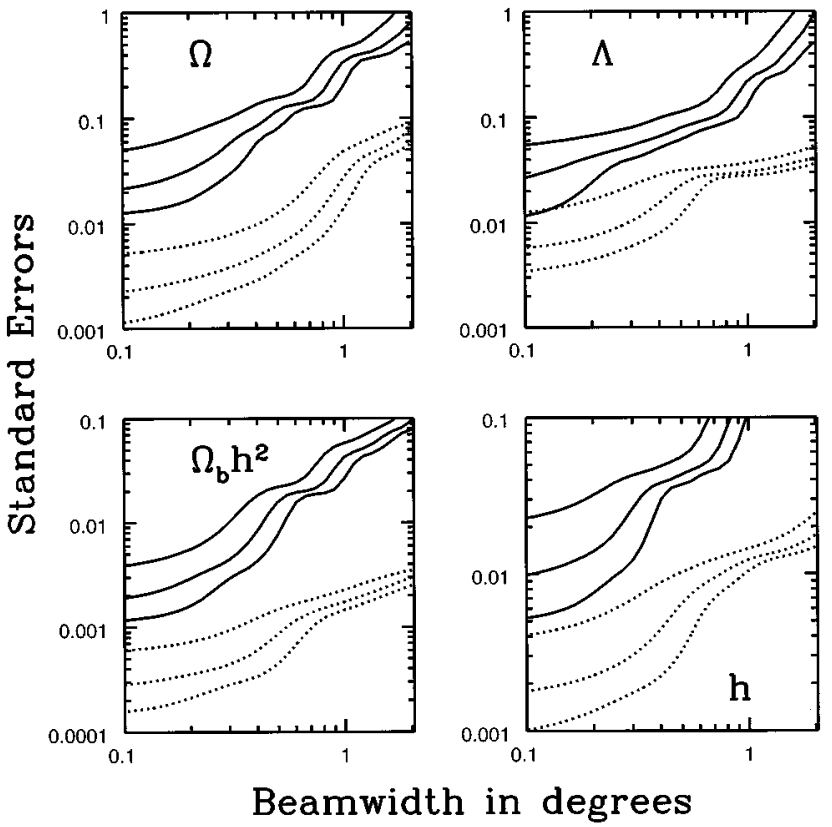

FIG. 3. The standard errors for $\Omega, \Lambda, \Omega_{b} h^{2}$, and $h$ that can be obtained with a full-sky mapping experiment as a function of the beam width $\theta_{\text {FWHM }}$ for noise levels $w^{-1}=2 \times 10^{-15}, 9 \times 10^{-15}$, and $4 \times 10^{-14}$ (from lower to upper curves). The underlying model is "standard CDM." The solid curves are the sensitivities attainable with no prior assumptions about the values of any of the other cosmological parameters. The dotted curves are the sensitivities that would be attainable assuming that all other cosmological parameters, except the normalization $(Q)$, were fixed. The results for a mapping experiment which covers only a fraction $f_{\text {sky }}$ of the sky can be obtained by scaling by $f_{\text {sky }}^{-1 / 2}$ [cf., Eq. (20)].

assumptions. First, we consider the case where none of the parameters are known. Then we consider the results under the most optimistic assumption that all of the other parameters, except the normalization (which will never be determined more accurately by any other observations), are fixed. Realistically, prior information on some of the parameters will be available, and so the standard errors will fall between these two extremes.

Figures 3 and 4 show the standard errors for various parameters that can be obtained with a full-sky mapping experiment as a function of the beam width $\theta_{\mathrm{FWHM}}$ for noise levels $w^{-1}=2 \times 10^{-15}, 9 \times 10^{-15}$, and $4 \times 10^{-14}$ (from lower to upper curves). The underlying model is "standard CDM." The solid curves are the sensitivities attainable with no prior assumptions about the values of any of the other cosmological parameters. The dotted curves are the sensitivities that would be attainable assuming that all other cosmological parameters, except the normalization $(Q)$, were fixed. The analogous results for a mapping experiment which covers only a fraction $f_{\text {sky }}$ of the sky can be obtained by scaling by $f_{\text {sky }}^{-1 / 2}$ [cf., Eq. (20)].

\section{A. Total density and cosmological constant}

The results for $\Omega$ were discussed in Ref. [31]. From the $\Omega$ panel in Fig. 3, it should be clear that a CMB mapping experiment with subdegree resolution could potentially de- 


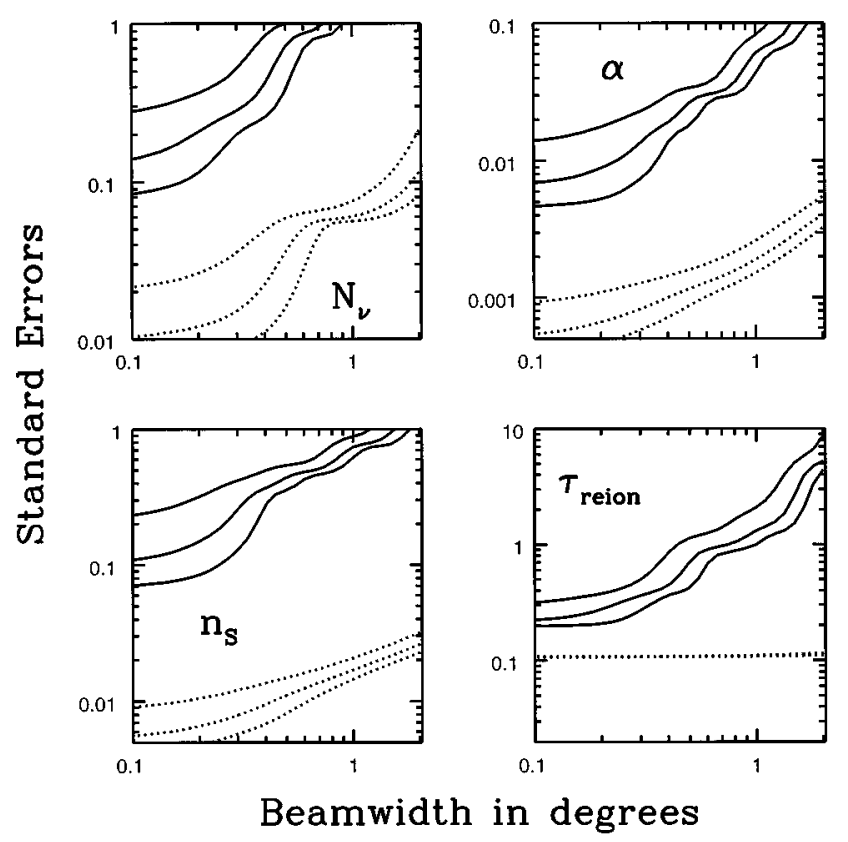

FIG. 4. Like Fig. 3, but for $\alpha, N_{\nu}, \tau_{\text {reion }}$, and $n_{S}$.

termine $\Omega$ to better than $10 \%$ with minimal assumptions, and perhaps better than $1 \%$ with prior information on other cosmological parameters. This would be far more precise than any conventional measurement of $\Omega$. Furthermore, unlike mass inventories which measure only the matter density $\Omega_{0}$, this measurement includes the contribution to the density from a cosmological constant (i.e., vacuum energy) and, therefore, directly probes the geometry of the Universe. This determination follows from the angular location of the first Doppler peak. Therefore, our results show that if the Doppler peak is found to be at $l \simeq 200$, it will suggest a value of $\Omega=1$ to within a few percent of unity. This result will be independent of the values of other cosmological parameters and will therefore be the most precise test for the flatness of the Universe and thus a direct test of the inflationary hypothesis. Numerical calculations suggest that the effect of geometry on the CMB spectrum may be slightly more dramatic than indicated by our semianalytic algorithm. If so, our final results on the sensitivity to $\Omega$ are a conservative estimate.

The sensitivity to $\Lambda$ is similar. Currently, the strongest bounds to the cosmological constant come from gravitational-lensing statistics [37] which only constrain $\Lambda$ to be less than 0.5. Measurement of the deceleration parameter $q_{0}=\Omega_{0} / 2-\Lambda$ could provide some information on $\Lambda$, but the measurements are tricky, and the result will depend on the matter density. On the other hand, a CMB mapping experiment should provide a measurement of Lambda to better than \pm 0.1 , which will easily distinguish between a $\Lambda$-dominated Universe and either an open or flat matterdominated Universe.

\section{B. Baryon density and Hubble parameter}

The current range for the baryon-to-photon ratio allowed by big-bang nucleosynthesis (BBN) is $0.0075 \leq \Omega_{b} h^{2}$ $\lesssim 0.024$ [38]. This gives $\Omega_{b} \lesssim 0.1$ for the range of acceptable values of $h$, which implies that if $\Omega=1$, as suggested by inflationary theory (or even if $\Omega \gtrsim 0.3$ as suggested by cluster dynamics), then the bulk of the mass in the Universe must be nonbaryonic. On the other hand, x-ray-cluster measurements might be suggesting that the observed baryon density is too high to be consistent with BBN [39]; this becomes especially intriguing given the recent measurement of a large primordial deuterium abundance in quasar absorption spectra [40]. The range in the BBN prediction can be traced primarily to uncertainties in the primordial elemental abundances. There is, of course, also some question as to whether the x-raycluster measurements actually probe the universal baryon density. Clearly, it would be desirable to have an independent measurement of $\Omega_{b} h^{2}$. The $\Omega_{b} h^{2}$ panel in Fig. 3 shows that the CMB should provide such complementary information. The implications of CMB maps for the baryon density depend quite sensitively on the experiment. As long as $\theta_{\text {FWHM }} \leq 0.5$, the CMB should (with minimal assumptions) at least be able to rule out a baryon-dominated Universe $\left(\Omega_{b} \gtrsim 0.3\right)$ and therefore confirm the predictions of BBN. With angular resolutions that approach $0.1^{\circ}$ (which might be achievable, for example, with a ground-based interferometry map [41] to complement a satellite map), a CMB map would provide limits to the baryon-to-photon ratio that were competitive with BBN. Furthermore, if other parameters can be fixed, the CMB might be able to restrict $\Omega_{b} h^{2}$ to a small fraction of the range currently allowed by BBN.

Current state-of-the-art measurements of the Hubble parameter approach precisions of roughly $10 \%$, and as a result of systematic uncertainties in the distance ladder, it is unlikely that any determinations in the foreseeable future will be able to improve upon this result. The panel for $h$ in Fig. 3 shows that, even with minimal assumptions, a mapping experiment with angular resolution better than $0.5^{\circ}$ will provide a competitive measurement; with additional assumptions, a much more precise determination is possible. It should also be noted that the CMB provides a measurement of the Hubble parameter which is entirely independent of the distance ladder or any cosmological distance determination.

As a technical aside, we mention that in calculating the curvature matrix, Eq. (22), we choose $\Omega_{0} h^{2}$ as an independent parameter instead of $h$, and then transform the curvature matrix back to the displayed parameters. The reason for this choice is that the power spectrum depends on $h$ only indirectly through the quantities $\Omega_{0} h^{2}$ and $\Omega_{b} h^{2}$, and the linear approximation to the change in the spectrum in Eq. (22) is more accurate for the parameter $\Omega_{0} h^{2}$. This parameter choice also explicitly accounts for the approximate degeneracy between models with the same value of $\Omega_{0} h^{2}$ but differing $\Lambda$ [30].

\section{Reionization}

As discussed above, the effects of reionization can be quantified, to a first approximation, by $\tau_{\text {reion }}$, the optical depth to the surface of last scatter, and there are several arguments which suggest $\tau_{\text {reion }} \leqslant 1$ [19]. First of all, significant reionization would lead to anisotropies on arcminute scales due to the Vishniac effect [28] or to spectral (Compton- $y$ ) distortions of the CMB [42]. Order-ofmagnitude estimates for the values of $\tau_{\text {reion }}$ expected in adiabatic models based on Press-Schechter estimates of the frac- 
tion of mass in collapsed objects as a function of redshift suggest that $\tau_{\text {reion }}$ is probably less than unity $[19,43]$. Moreover, the numerous detections of anisotropy at the degree scale [2] also show an absence of excessive reionization. Assuming complete reionization at a redshift $z_{\text {reion }}$, the optical depth with our standard-CDM values is $\tau_{\text {reion }} \simeq 0.001 z_{\text {reion }}^{3 / 2}$, and so $\tau_{\text {reion }} \lesssim 1$ corresponds to $z_{\text {reion }}$ $\lesssim 100$.

The $\tau_{\text {reion }}$ panel of Fig. 4 illustrates that, with minimal assumptions, any map with subdegree angular resolution will probe the ionization history (i.e., $z_{\text {reion }} \leqslant 1000$ ), and maps with resolutions better than a half degree can restrict the optical depth to 0.5 or less. While different ionization histories with the same total optical depth can give different power spectra, as long as the reionization is not too severe, simple damping of the primary anisotropies is always the dominant effect. The lower curves, assuming other parameters are fixed except for $Q$, are flat because at scales smaller than $2^{\circ}$, the effects of $\tau_{\text {reion }}$ are precisely degenerate with a shift in $Q$. The lower curves nearly coincide for the different noise levels because all of the leverage in distinguishing $\tau_{\text {reion }}$ comes at low $l$ where the degeneracy with $Q$ is broken, and at these scales the cosmic variance dominates the measurement errors.

Although temperature maps alone may not provide a stringent probe of the ionization history, polarization maps may provide additional constraints. The polarization produced at recombination is generally small, but that produced during reionization can be much larger. Heuristically, the temperature anisotropy which is damped by reionization goes into polarization. Therefore, it is likely that polarization maps will be able to better constrain $\tau_{\text {reion }}$ when used in conjunction with temperature maps.

\section{Neutrinos}

We have also investigated the sensitivity of CMB anisotropies to $N_{\nu}$, the effective number of neutrino degrees of freedom at decoupling. The number of noninteracting relativistic species affects the CMB spectrum by changing the time of matter-radiation equality, although this cannot be distinguished from the same effect due to changes in $h$, $\Omega_{0}$, and $\Lambda$. However, neutrinos (and other noninteracting degrees of freedom which are relativistic at decoupling) free stream and therefore have a unique effect on the growth of potential perturbations. This will be reflected in the detailed shape of the CMB spectrum, especially from the contribution of the early-time ISW effect. In standard CDM, there are the three light-neutrino species. However, some particle-physics models predict the existence of additional very light particles which would exist in abundance in the Universe. Furthermore, if one of the light neutrinos has a mass greater than an $\mathrm{eV}$, as suggested by mixed dark-matter models [44] and possibly by the Los Alamos experiment [45], then it would be nonrelativistic at decoupling, and so the effective number of neutrinos measured by the CMB would $\mathrm{be}^{2} N_{\nu}<3$. These

\footnotetext{
${ }^{2}$ In such a case, the massive neutrino would have additional effects on the CMB [6]. Although we have not included these effects, our analysis still probes variations in $N_{\nu}$, and our results are suggestive of the sensitivity of CMB anisotropies to a massive neutrino.
}

limits would be similar to limits on the number of relativistic noninteracting species from BBN. However, at the time of BBN, any particle with mass less than $1 \mathrm{MeV}$ would be relativistic, whereas at decoupling, only those with masses less than $1 \mathrm{eV}$ would be relativistic, and so the quantities probed by $\mathrm{BBN}$ and by the $\mathrm{CMB}$ are somewhat different.

The panel for $N_{\nu}$ in Fig. 4 shows the sensitivity of CMB anisotropies to variations in the effective number of noninteracting nonrelativistic species at decoupling. When one takes into account systematic uncertainties in primordial elemental abundances, BBN constrains the effective number of relativistic (i.e., less than a few $\mathrm{MeV}$ ) neutrino species to be less than 3.9 [38]. Figure 1 illustrates that any mapping experiment with angular resolution better than $0.5^{\circ}$ should provide complementary information; if other parameters can be determined or constrained, then the CMB has the potential to provide a much more precise probe of the number of light neutrinos at the decoupling epoch.

\section{E. Inflationary observables}

We have also studied the precision with which the inflationary observables $n_{S}, n_{T}$, and $r$ can be probed. Inflation predicts relations between the scalar spectral index $n_{S}$, the tensor spectral index $n_{T}$, and the ratio $r$ [46]. Therefore, precise measurement of these parameters provides a test of inflationary cosmology and perhaps probes the inflaton potential [47].

Knox [32] performed a Monte Carlo calculation to address the question of how accurately CMB anisotropies can measure the inflationary observables assuming all other cosmological parameters were known. Here, we generalize the results to a broader range of pixel noises and beam widths and take into account the uncertainties in all other cosmological parameters through the covariance matrix.

In Fig. 5, we show the standard errors on the inflationary observables that could be obtained with mapping experiments with various levels of pixel noise and beam widths. The parameters of the underlying model used here are the same "standard-CDM" parameters used in Figs. 3 and 4, except here we set $r=Q_{T}^{2} / Q_{S}^{2}=0.28, \quad n_{S}=0.94$, and $n_{T}=-0.04$. We do so for two reasons: First, the tensor spectral index is unconstrained without a tensor contribution; second, these parameters will facilitate comparison with the results of Ref. [32]. The solid curves are the standard errors that would be obtained with no assumptions about the values of these or any other of the cosmological parameters. The dotted curves are the standard errors that would be attainable by fitting to only these four inflationary observables and assuming all other cosmological parameters are known. (Note that this differs from the dotted curves in Figs. 3 and 4.)

The dotted curves in Fig. 5 with a beam width of $0.33^{\circ}$ are in good numerical agreement with the results of Ref. [32]. This verifies that the covariance-matrix and Monte Carlo calculations agree. Next, note that unless the other cosmological parameters can be determined (or are fixed by assumption), the results of Ref. [32] for the sensitivities of CMB anisotropy maps to the inflationary observables are very optimistic. In particular, temperature maps will be un- 


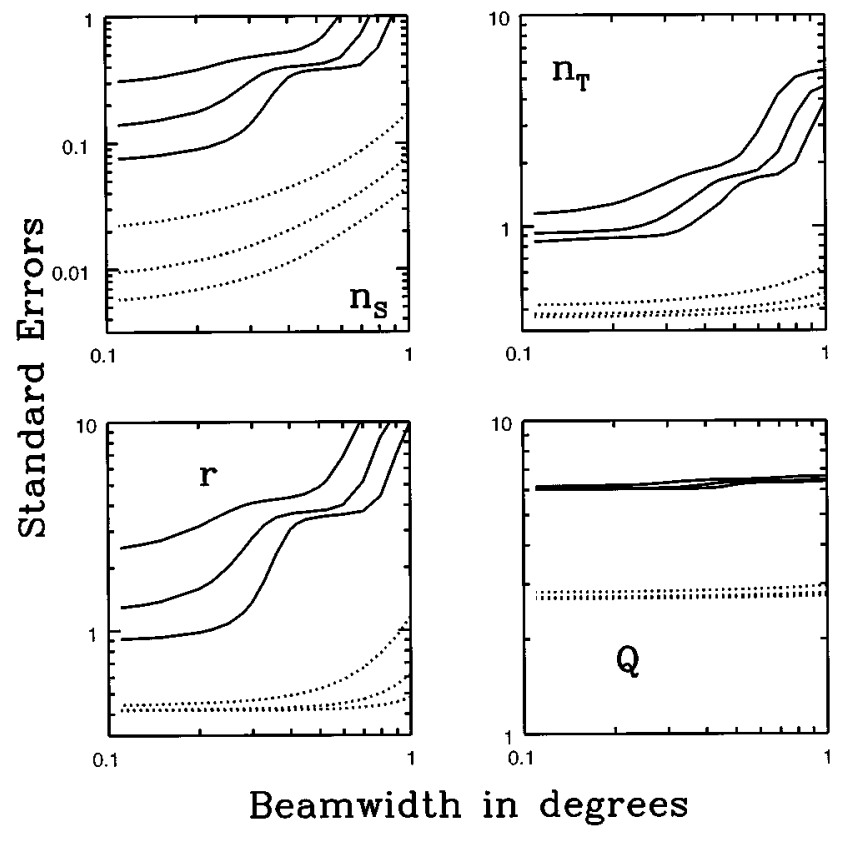

FIG. 5. The standard errors on the inflationary observables $n_{S}, n_{T}, r=Q_{T}^{2} / Q_{S}^{2}$, and $Q$ that can be obtained with a full-sky mapping experiment as a function of the beam width $\theta_{\mathrm{FWHM}}$ for noise levels $w^{-1}=2 \times 10^{-15}, 9 \times 10^{-15}$, and $4 \times 10^{-14}$ (from lower to upper curves). The parameters of the underlying model are the "standard $=$ CDM" values, except we have set $r=0.28, n_{S}=0.94$, and $n_{T}=-0.04$. The solid curves are the sensitivities attainable with no prior assumptions about the values of any of the other cosmological parameters. The dotted curves are the standard errors that would be attainable by fitting to only these four inflationary observables and assuming all other cosmological parameters are known. (Note that this differs from the dotted curves in Fig. 4.) The results for a mapping experiment which covers only a fraction $f_{\text {sky }}$ of the sky can be obtained by scaling by $f_{\text {sky }}^{-1 / 2}$ [cf., Eq. (20)].

able to provide any useful constraint to $r$ and $n_{T}$ (and it will be impossible to reconstruct the inflaton potential) unless the other parameters can be measured independently. However, if the classical cosmological parameters can be determined by other means (or fixed by assumption), the dotted curves in Fig. 5 show that fairly precise information about the inflaton potential will be attainable. CMB polarization maps may provide another avenue towards improved determination of the inflationary observables [48].

The flatness of the dotted curves for $r$ and $n_{T}$ in Fig. 5 is due to the fact that the contribution of the tensor modes to the CMB anisotropy drops rapidly on angular scales smaller than roughly $1^{\circ}$. The solid curves decrease with $\theta_{\text {FWHM }}$ because the other cosmological parameters (e.g., $\Omega_{b} h^{2}$ and $h$ ) become determined with much greater precision as the angular resolution is improved.

Of course, the precision with which the normalization of the perturbation spectrum can be measured with $\mathrm{CMB}$ anisotropies (even current COBE measurements) is-and will continue to be-unrivaled by traditional cosmological observations. Galactic surveys probe only the distribution of visible mass, and the distribution of dark matter could be significantly different (this is the notion of biasing). The dotted figures in the panel for $Q$ in Fig. 5 are the sensitivities that would be obtained assuming all other parameters were known. This standard error would be slightly larger if there were no tensor modes included, because as $r$ is increased (with the overall normalization $Q$ held fixed), the scalar contribution is decreased. Therefore, tensor modes decrease the anisotropy on smaller angular scales, the signal to noise is smaller, and the sensitivity to $Q$ (and other parameters) is slightly decreased. The effect of variations in other underlying-model parameters on our results is discussed further below.

\section{F. What if the underlying model is different?}

Now we consider what might be expected if the underlying theory differs from that assumed here. Generally, the parameter determination will be less precise in models in which there is less cosmological anisotropy, as reflected in Eq. (20).

What happens if the normalization differs from the central COBE value we have adopted here? The normalization raises or lowers all the multipole moments; therefore, from Eq. (20), the effect of replacing $Q$ with $Q^{\prime}$ is equivalent to replacing $w^{-1}$ with $w^{-1}\left(Q / Q^{\prime}\right)$. In Figs. 3, 4, and 5, the solid curves, which are spread over values of $w^{-1}$ that differ by more than an order of magnitude, are all relatively close. On the other hand, the uncertainty in the COBE normalization is $10 \%$. Therefore, our results are insensitive to the uncertainty in the normalization of the power spectrum.

If there is a significant contribution to the COBE-scale anisotropy from tensor modes, then the normalization of the scalar power spectrum is lower, the Doppler peaks will be lower, and parameter determinations that depend on the Doppler-peak structure will be diluted accordingly. On the other hand, the tensor spectral index, which is important for testing inflationary models, will be better determined.

Similarly, reionization damps structure on Doppler-peak angular scales, and so if there is a significant amount of reionization, then much of the information in the CMB will be obscured. On the other hand, there are several indications summarized above that damping due to reionization is not dramatic. In Ref. [31], we displayed (in Fig. 2 therein) results for the standard error in $\Omega$ for a model with $\tau_{\text {reion }}=0.5$. As expected, the standard error is larger (but by no more than a factor of 2) than in a model with no reionization.

If $\Lambda$ is nonzero, $h$ is small, or $\Omega_{b} h^{2}$ is large, then the signal to noise should increase and there will be more information in the CMB. If the scalar spectral index is $n_{S}>1$, then the Doppler peaks will be higher, but in the more likely case (that predicted by inflation), $n_{S}$ will be slightly smaller than unity. This would slightly decrease the errors.

If $\Omega$ is less than unity, then the Doppler peak (and all the information encoded therein) is shifted to smaller angular scales. Thus one might expect parameter determinations to become less precise if $\Omega<1$. By explicit numerical calculation, we find that for $\Omega=0.5$ (with all other parameters given by the "standard-CDM" values), our estimates for the standard error for $\Omega$ is at the same level as the estimate we obtained for $\Omega=1$. Therefore, even if $\Omega$ is as small as 0.3 , our basic conclusions that $\Omega$ can be determined to \pm 0.1 with minimal assumptions are valid. The sensitivity to some of 

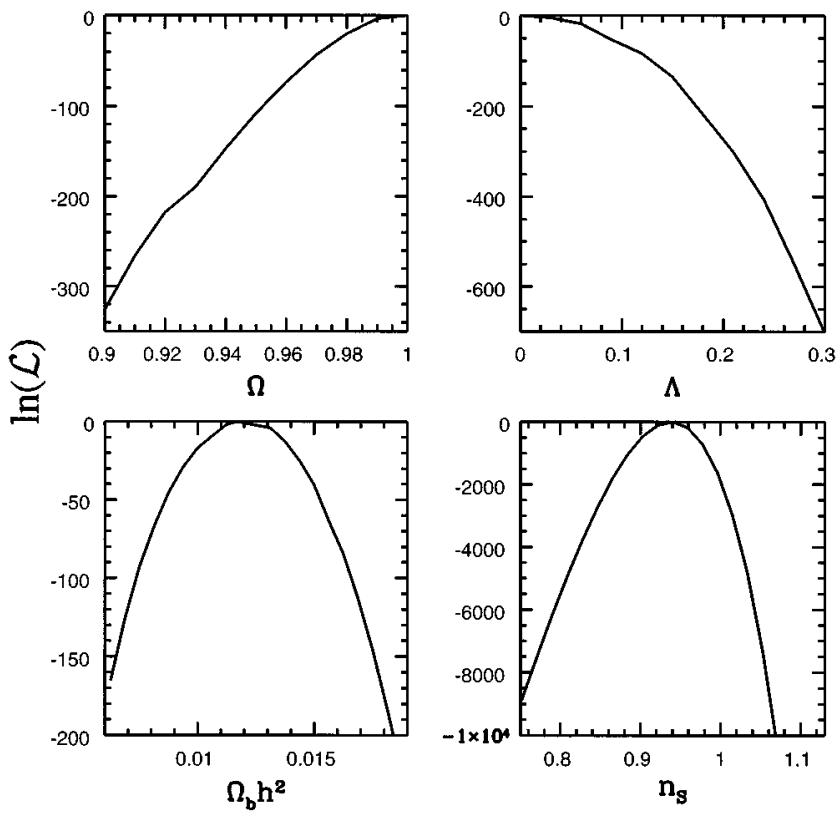

FIG. 6. Plots of the $\log$ likelihood as a function of $\Omega, \Lambda$, $\Omega_{b} h^{2}$, and $n_{S}$ for the "standard-CDM" model with tensor modes (so $n_{S}=0.94$ and $r=0.28$ ). Note that the log likelihood looks parabolic.

the other parameters, in particular $h$, is (not surprisingly) significantly degraded in an open Universe.

\section{GAUSSIAN APPROXIMATION TO THE LIKELIHOOD FUNCTION}

It is an implicit assumption of the covariance-matrix analysis that the likelihood function has an approximate Gaussian form within a sufficiently large neighborhood of the maximum-likelihood point. Equation (21) only approximates the likelihood function in a sufficiently small neighborhood around the maximum. The detailed functional form of the likelihood function is given in Ref. [32]. If the likelihood function fails to be sufficiently Gaussian near the maximum, then the covariance-matrix method is not guaranteed to produce an estimate for the standard error, and a more involved (Monte Carlo) analysis would be essential. Therefore, in the following we indicate the applicability of the Gaussian assumption for the likelihood.

First, we note that our parametrization has the property that the individual parameters are approximately independent. This is suggested by direct examination of the eigenvectors of the covariance matrix. This is also supported by preliminary Monte Carlo results. Therefore it is simplest to examine the behavior of the likelihood as a function of individual parameters in order to determine if a parabolic approximation to $\ln (\mathcal{L})$ (the log likelihood) is admissible.

In Fig. 6, we display the dependence of $\ln (\mathcal{L})$ on several parameters of interest. In this example, we used $w^{-1}=9 \times 10^{-15}$ and $\theta_{\mathrm{FWHM}}=0.25$. As is clear from this figure, the functional forms are well fit by parabolic approximations, within regions of size $\sim 3 \sigma$ around the maximum point. This is sufficient to apply the covariance-matrix analysis to the determination of the standard errors, and our analysis above is justified.
Although we have not done an exhaustive survey of the likelihood contours in the 11-dimensional parameter space, Fig. 6 also suggests that there are no local maxima anywhere near the true maximum. Therefore, fitting routines will probably not be troubled by local maxima. This also suggests, then, that there will be no degeneracy between various cosmological models with a CMB map (in contrast to the conclusions of Ref. [30]), unless the models are dramatically different. In this event (which we consider unlikely), one would then be forced to choose between two quite different models, and it is probable that additional data would determine which of the two models is correct.

\section{CONCLUSIONS}

We have used a covariance-matrix approach to estimate the precision with which 11 cosmological parameters of interest could be determined with a CMB temperature map. We used realistic estimates for the pixel noise and angular resolution and quantified the dependence on the assumptions about various cosmological parameters that would go into the analysis. The most interesting result is for $\Omega$ : With only the minimal assumption of primordial adiabatic perturbations, proposed CMB satellite experiments [3] could potentially measure $\Omega$ to $5 \%$. With prior information on the values of other cosmological parameters possibly attainable in the forthcoming years, $\Omega$ might be determined to better than $1 \%$. This would provide an entirely new and independent determination of $\Omega$ and would be far more accurate than the values given by any traditional cosmological observations. Furthermore, typical mass inventories yield only the matter density. Therefore, they tell us nothing about the geometry of the Universe if the cosmological constant is nonzero. A generic prediction of inflation is a flat Universe; therefore, locating the Doppler peak will provide a crucial test of the inflationary hypothesis.

CMB temperature maps will also provide constraints on $\Lambda$ far more stringent than any current ones, and will provide a unique probe of the inflationary observables. Information on the baryon density and Hubble constant will complement and perhaps even improve upon current observations. Furthermore, although we have yet to include polarization maps in our error estimates, it is likely that they will provide additional information, at least regarding ionization history.

We have attempted to display our results in a way that will be useful for future CMB experimental design. Although a satellite mission offers the most promising prospect for making a high-resolution CMB map, our error estimates should also be applicable to complementary balloon-borne or ground-based experiments which map a limited region of the sky. The estimates presented here can also be used for a combination of complementary experiments.

Although we have been able to estimate the precision with which CMB temperature maps will be able to determine cosmological parameters, there is still much theoretical work that needs to be done before such an analysis can realistically be carried out. To maximize the likelihood in a multidimensional parameter space, repeated evaluation of the $\mathrm{CMB}$ spectrum for a broad range of model parameters is needed. Therefore, quick and accurate calculations of the CMB anisotropy spectrum will be crucial for the data analysis. Sev- 
eral independent numerical calculations of the CMB anisotropy spectrum now agree to roughly $1 \%$ [49]. However, these calculations typically require hours of workstation time per spectrum and are therefore unsuitable for fitting data. We have begun to extend recent analytic approximations to the CMB spectra $[9,50]$ with the aim of creating a highly accurate and efficient power spectrum code. Our current code evaluates spectra in a matter of seconds on a workstation, though our calculations are not yet as accurate as the full numerical computations, except in a limited region of parameter space. It is likely, however, that the analytic results can be generalized with sufficient accuracy.

The other necessary ingredient will be an efficient and reliable likelihood-maximization routine. Preliminary fits to simulated data with a fairly simple likelihood-maximization algorithm suggest that the cosmological parameters can indeed be reproduced with the precision estimated here [8].

In summary, our calculations indicate that CMB temperature maps with good angular resolution can provide an unprecedented amount of quantitative information on cosmological parameters. These maps will also inform us about the origin of structure in the Universe and test ideas about the earliest Universe. We hope that these results provide additional impetus for experimental efforts in this direction.

\section{ACKNOWLEDGMENTS}

We would like to thank Scott Dodelson, Lloyd Knox, Michael Turner, and members of the MAP Collaboration for helpful discussions, and George Smoot for useful comments. Martin White graciously provided Boltzmann-code power spectra for benchmarking our code. This work was supported in part by the (U.S.) DOE under Contracts No. DEFG0292-ER 40699 at Columbia University and No. DEFG0285-ER 40231 at Syracuse University, by the Harvard Society of Fellows, by the NSF under Contract No. ASC 93-18185 (GC3 collaboration) at Princeton University, by NASA under Contracts No. NAG5-3091 at Columbia University and No. NAGW-2448, and under the MAP Mission Concept Study Proposal at Princeton University. Portions of this work were completed at the Aspen Center for Physics.
[1] G. F. Smoot et al., Astrophys. J. Lett. 396, L1 (1992).

[2] For a review of CMB measurements through the end of 1994, see M. White, D. Scott, and J. Silk, Annu. Rev. Astron. Astrophys. 32, 329 (1994).

[3] See, e.g., C. L. Bennett et al. and M. A. Janssen et al., NASA Mission Concept Study Proposals; F. R. Bouchet et al., report, astro-ph/9507032, 1995 (unpublished).

[4] R. G. Crittenden and N. Turok, Phys. Rev. Lett. 75, 2642 (1995); A. Albrecht et al., report, astro-ph/9505030, 1995 (unpublished); R. Durrer, A. Gangui, and M. Sakellariadou, Phys. Rev. Lett. 76, 579 (1996); B. Allen et al., in CMB Anisotropies Two Years After COBE: Observations, Theory and the Future, Proceedings of the CWRU Workshop, edited by L. M. Krauss (World Scientific, Singapore, 1994); J. Magueijo et al., Phys. Rev. Lett. 76, 2617 (1996).

[5] W. Hu, E. F. Bunn, and N. Sugiyama, Astrophys. J. Lett. 447, 59 (1995).

[6] S. Dodelson, E. Gates, and A. Stebbins, Astrophys. J. (to be published); E. Bertschinger and P. Bode (private communication).

[7] J. C. Mather et al., Astrophys. J. Lett. 420, 439 (1994).

[8] D. N. Spergel, in Dark Matter, Proceedings of the conference, College Park, Maryland, 1994, edited by S. S. Holt and C. L. Bennett (AIP, New York, 1995).

[9] W. Hu and N. Sugiyama, Phys. Rev. D 51, 2599 (1995); Astrophys. J. 444, 489 (1995).

[10] E. Bertschinger, report, astro-ph/9506070 (unpublished).

[11] J. Silk, Astrophys. J. 151, 459 (1968).

[12] M. Zaldarriaga and D. D. Harari, Phys. Rev. D 52, 3276 (1995).

[13] M. S. Turner, M. White, and J. E. Lidsey, Phys. Rev D 48, 4613 (1993).

[14] Y. Wang, Phys. Rev. D 53, 639 (1996).

[15] A. Kosowsky and M. S. Turner, Phys. Rev. D 52, 1739 (1995).

[16] L. A. Kofman and A. A. Starobinsky, Sov. Astron. Lett. 11 , 271 (1986).
[17] M. Kamionkowski and D. N. Spergel, Astrophys. J. 432, 7 (1994).

[18] W. Hu and N. Sugiyama, Astrophys. J. (to be published).

[19] M. Kamionkowski, D. N. Spergel, and N. Sugiyama, Astrophys. J. Lett. 426, L57 (1994).

[20] D. H. Lyth and E. D. Stewart, Phys. Lett. B 252, 336 (1990); B. Ratra and P. J. E. Peebles, Phys. Rev. D 52, 1837 (1995); M. Bucher, A. S. Goldhaber, and N. Turok, ibid. 52, 3314 (1995); K. Yamamoto, M. Sasaki, and T. Tanaka, Astrophys. J. 455, 412 (1995).

[21] M. Kamionkowski, B. Ratra, D. N. Spergel, and N. Sugiyama, Astrophys. J. Lett. 434, L1 (1994).

[22] N. Kaiser, Astrophys. J. 282, 374 (1984).

[23] W. Hu and M. White, Astron. Astrophys. (to be published).

[24] M. Tegmark, in Proceedings of the International School of Physics “Enrico Fermi,', Course CXXXII, Varenna, 1995 (in press).

[25] See, e.g., U. Seljak, Astrophys. J. 463, 1 (1996).

[26] M. J. Rees and D. W. Sciama, Nature (London) 517, 611 (1968).

[27] R. Sunyaev and Ya. B. Zeldovich, Annu. Rev. Astron. Astrophys. 18, 537 (1980).

[28] J. P. Ostriker and E. T. Vishniac, Astrophys. J. 306, 51 (1986); E. T. Vishniac, ibid. 322, 597 (1987).

[29] F. Persi et al., Astrophys. J. 442, 1 (1995).

[30] J. R. Bond et al., Phys. Rev. Lett. 72, 13 (1994).

[31] G. Jungman, M. Kamionkowski, A. Kosowsky, and D. N. Spergel, Phys. Rev. Lett. 76, 1007 (1996).

[32] L. Knox, Phys. Rev. D 52, 4307 (1995).

[33] J. Magueijo and M. P. Hobson, report, astro-ph/9602023 (unpublished); M. P. Hobson and J. Magueijo, report, astro-ph/ 9603064 (unpublished).

[34] A. Gould, Astrophys. J. Lett. 440, 510 (1995).

[35] G. Hinshaw, C. L. Bennett, and A. Kogut, Astrophys. J. Lett. 441, L1 (1995). 
[36] K. M. Górski et al., Astrophys. J. Lett. 430, L89 (1994); C. L. Bennett et al., Astrophys. J. 436, 423 (1994).

[37] See, e.g., C. Kochanek, in Dark Matter [8], and references therein.

[38] C. J. Copi, D. N. Schramm, and M. S. Turner, Phys. Rev. Lett. 75, 3981 (1995).

[39] U. G. Briel, J. P. Henry, and H. Bohringer, Astron. Astrophys. 259, L31 (1992); S. D. M. White et al., Nature (London) 366, 429 (1993); G. Steigman and J. E. Felten, in Proceedings of the St. Petersburg Gamow Seminar, St. Petersburg, Russia, 1994, edited by A. M. Bykov and A. Chevalier, Space Sci. Rev. (Kluwer, Dordrecht, in press).

[40] A. Songalia et al., Nature (London) 368, 599 (1994); M. Rugers and C. J. Hogan, Astrophys. J. Lett. (to be published).

[41] S. T. Myers (private communication).

[42] Ya. B. Zeldovich and R. A. Sunyaev, Astrophys. Space Sci. 4, 301 (1969); J. C. Mather et al., Astrophys. J. Lett. 354, L37 (1990).
[43] M. Tegmark, J. Silk, and A. Blanchard, Astrophys. J. 420, 484 (1994).

[44] J. A. Holtzman, Astrophys. J. Suppl. 71, 1 (1989); M. Davis, F. J. Summers, and D. Schlegel, Nature (London) 359, 393 (1992); J. R. Primack and J. A. Holtzman, Astrophys. J. 405, 428 (1993); A. Klypin et al., ibid. 416, 1 (1993).

[45] LSND Collaboration, C. Athanassopoulos et al., Phys. Rev. Lett. 75, 2650 (1995).

[46] A. R. Liddle and D. Lyth, Phys. Lett. B 291, 391 (1992).

[47] J. E. Lidsey et al., report, astro-ph/9508078 (unpublished).

[48] R. G. Crittenden, D. Coulson, and N. G. Turok, Phys. Rev. D 52, 5402 (1995); R. Crittenden, R. L. Davis, and P. J. Steinhardt, Astrophys. J. Lett. 417, L13 (1993); D. Coulson, R. G. Crittenden, and N. G. Turok, Phys. Rev. Lett. 73, 2390 (1994).

[49] COMBA Collaboration, P. J. Steinhardt et al. (private communication).

[50] U. Seljak, Astrophys. J. Lett. 435, L87 (1994). 